\title{
A phase II study of neoadjuvant bevacizumab plus capecitabine and concomitant radiotherapy in patients with locally advanced rectal cancer
}

\author{
Giampietro Gasparini - Francesco Torino - Takayuki Ueno • Stefano Cascinu • \\ Teresa Troiani - Alberto Ballestrero - Rossana Berardi - Junichi Shishido • \\ Akihiko Yoshizawa · Yukiko Mori · Satoshi Nagayama · Paola Morosini · \\ Masakazu Toi
}

Received: 25 August 2011/Accepted: 20 December 2011/Published online: 3 January 2012 (C) Springer Science+Business Media B.V. 2011

\begin{abstract}
Purpose To assess safety and activity of neoadjuvant bevacizumab, capecitabine and standard radiotherapy in locally advanced rectal cancer as well as potential predictive biomarkers.

Patients and methods The multicentric phase II study enrolled 43 patients who received bevacizumab infusion $(5 \mathrm{mg} / \mathrm{kg})$ every 2 weeks for 4 cycles; oral capecitabine at $825 \mathrm{mg} / \mathrm{m}^{2}$ twice a day for 5.5 weeks with external-beam irradiation (50.4 Gy in 28 fractions over 5.5 weeks). We determined certain biomarkers before and after therapy for correlation with response.
\end{abstract}

G. Gasparini $(\bowtie) \cdot$ F. Torino

Unità Operativa Complessa di Oncologia Medica, Azienda

Complesso Ospedaliero di Rilevanza Nazionale "S. Filippo

Neri”, Via G. Martinotti, 20, 00135 Rome, Italy

e-mail: gasparini.oncology@ hotmail.it

T. Ueno $\cdot$ J. Shishido $\cdot$ Y. Mori · S. Nagayama $\cdot$ M. Toi

Graduate School of Medicine, Kyoto University, Kyoto, Japan

S. Cascinu $\cdot$ R. Berardi

Oncologia Medica Università delle Marche-Ospedale Umberto I,

Ancona, Italy

T. Troiani

Oncologia Medica Università "Federico II", Napoli, Italy

\section{A. Ballestrero}

Dipartimento Medicina Interna Università di Genova, Genoa, Italy

\section{A. Yoshizawa}

Department of Laboratory Medicine,

Shinshu University Hospital, Nagano, Japan

P. Morosini

Medical Affairs-Oncology, Roche S.p.A., Milan, Italy
Results Post-operative histologic examination revealed no residual cancer cells in 6 of the 43 patients (14\%; $95 \%$ confidence limits 3.60-24.31\%). In another 22 patients $(51.2 \%)$ a varying percentage of cancer cells in residual areas of fibrosis/ necrosis was found, corresponding to Mandard TRG 2 or 3 classification. Tumor resection with negative circumferential margin was achieved in 38/40 $(95 \%)$ operated patients. Sphincter-sparing surgery was obtained in $31(72.1 \%)$ patients. Primary tumor and lymph nodes downstaging was observed in $15(34.9 \%)$ and 16 $(37.2 \%)$ cases, respectively. Neoadjuvant therapy was safe and well tolerated. The most frequent side effects were G1-2 diarrhea, proctitis, rectal bleeding and hypertension. No biomarker tested was significantly predictive of both pathological complete response and disease-free survival. Pre-treatment CD-34 vessel density, post-treatment Ki-67 labeling index and VEGFR-2 cancer cells expression significantly correlated with residual tumor area.

Conclusions The schedule of neoadjuvant therapy tested was safe and active. Pre-treatment vessel density by the panendothelial marker anti CD-34 antibody, post-treatment Ki-67 labeling index and VEGFR-2 expression were significantly associated to residual tumor area. The biomarkers correlations warrant further evaluation in prospective clinical trials.

Keywords Bevacizumab - Capecitabine - Radiotherapy · Rectal cancer $\cdot$ Neoadjuvant-treatment

\section{Introduction}

A number of studies and two meta-analyses demonstrated that in locally advanced rectal cancer (LARC) preoperative (neoadjuvant) radiation therapy significantly reduces the 
risk of local recurrence and cancer-specific mortality compared to surgery alone $[1,2]$. The addition of fluorouracil to radiation therapy significantly increases the rate of pathologic complete response (ypCR) and local control versus radiotherapy alone [3, 4] and chemoradiotherapy is now the standard of neoadjuvant treatment of LARC. The NSABP-R-03 trial compared neoadjuvant versus adjuvant chemoradiotherapy in LARC and demonstrated a significantly improved 5-years disease-free survival (DFS) favoring preoperative therapy, but not showing an overall survival (OS) advantage [5]. The identification of more effective radiation sensitizers represents a topical area of research in such a field. The aim is to improve local control and the possibility of preservation of sphincter function with the hope, ultimately, to avoid the need of abdominalperineal resection and permanent colostomy, thus improving the quality of life of patients.

Capecitabine is an oral fluoropyrimidine preferentially converted to the active metabolite within tumor cells with higher affinity on the enzyme thymidine phosphorylase [6]. It also showed similar activity compared to infusional fluorouracil when combined with radiotherapy [7].

Oxaliplatin is one of the most active single agents in the treatment of colorectal cancer and it is a potent radiosensitizing drug [8]. However, two recent controlled studies, the Prodiga 2-ACCORD 12/0405 trial and the STAR-01 trial did not demonstrate a statistically significant ypCR rate in the experimental arm with oxaliplatin compared to the standard fluoropyrimidine-based one $[9,10]$. Therefore, there was not a real therapeutic progress by using chemotherapy in LARC during the last decade [11].

Among the emerging strategies there are those based on the use of targeted therapies directed against the two more relevant targets in colorectal cancer, namely the epidermal growth factor receptor (EGFR) and vascular endothelial growth factor (VEGF). Phase I-II studies tested cetuximab, an $\mathrm{IgG}_{1}$ monoclonal antibody anti-EGFR in combination with chemotherapy and radiation therapy as neoadjuvant therapy in LARC [12]. However, in cohorts of unselected patients for K-ras cetuximab did not improve the ypCR rate [13].

More attractive appear the treatments directed to block VEGF. Bevacizumab, the FDA approved anti-VEGF antibody, significantly improved OS in patients with metastatic colorectal cancer when combined with irinotecan-based chemotherapy as first-line therapy [14]. More recently, bevacizumab was tested in the adjuvant setting in two phase III randomized trials in association with oxaliplatinbased chemotherapy. However, both the NSABP C-08 and AVANT studies failed to demonstrate a significant benefit in term of DFS and OS of bevacizumab plus chemotherapy versus chemotherapy alone $[15,16]$. Possible explanations for these discordant results in metastatic versus adjuvant settings may be related to the different schedules of chemotherapy used, the possible different biological context concerning the pattern of vascularity of primary tumors versus metastatic lesions and to the duration of administration of bevacizumab [15].

The use of anti-VEGF therapy in LARC is supported by: (1) the radiosensibilization activity demonstrated in experimental models [17] and the in vivo antivascular effects shown in human LARC in the studies performed by Willet et al. [18, 19].

Based on the above rationale it is presumed that LARC is characterized by different tumor biology as compared to radically resected early-stage colorectal cancer. Also the timing and dosing used of neoadjuvant bevacizumab may play a key role favoring its administration in this setting compared to the adjuvant one [15].

We performed the present phase II study in a series of patients affected by LARC in order to verify the toxicity and efficacy of the neoadjuvant schedule of bevacizumabcapecitabine combined with concomitant radiation therapy and to study the role of certain biomarkers in assessing response and outcome of such a therapy.

For the translational study aimed to identify potential predictive biomarkers we evaluated biomarkers related to microvessel density (MVD) and expression of vascular endothelial growth factor receptor-2 (VEGFR-2) as parameters of angiogenesis, tumor associated macrophages (CD68 antibody) apoptosis (M30 antibody), cell kinetics (anti-Ki-67 labeling index), as well as anti-thymidine synthase (TS) and anti-thymidine phosphorylase (TP) being targets of fluoropyrimidines.

\section{Patients and methods}

The ML18522 study (NCT01227707; clinicaltrial.gov) was approved by the Ethics Committee at the San Filippo Neri Hospital of Rome and by all the other participating Institutions. Each patient gave his/her written informed consent before being accrued.

\section{Eligibility criteria}

Patients $>18$ years old with histopathologically confirmed rectal adenocarcinoma with the inferior margin within $16 \mathrm{~cm}$ from the anal verge as assessed by rectosigmoidoscopy. The tumor had to have evidence of T2 disease with positive locoregional lymph nodes or T3/T4 disease by endorectal ultrasound, computed tomography (CT) and magnetic resonance imaging of the pelvis. Further inclusion criteria were Eastern Cooperative Oncology Group (ECOG) performance status $<2$ and adequate hematologic, liver and renal functions (neutrophils $\geq 1.5 \times 10^{9} / 1$, 
platelet count $\geq 100 \times 10^{9} /$; t total bilirubin concentration $<1.5 \times$ the upper normal limit (UNL); liver transaminases or alkaline phosphatase concentrations $<2.5 \times$ the UNL; serum creatinine $\leq 1.5 \times \mathrm{UNL}$ or creatinine clearance $>50 \mathrm{ml} / \mathrm{min}$, urine dipstick of proteinuria $<2+$ or $\leq 1 \mathrm{~g}$ of protein in $24 \mathrm{~h}$ urine).

\section{Exclusion criteria}

Metastatic disease, previous chemotherapy and/or radiation therapy and history of previous other cancers. Patients suffering from the following conditions were also ineligible: inflammatory bowel disease, malabsorption syndrome, uncontrolled hypertension, clinically significant cardiovascular disease (e.g. cerebrovascular stroke or myocardial infarction within 6 months), unstable angina, New York Heart Association (NYHA) grade II or greater congestive heart failure, cardiac arrhythmia requiring medication, bleeding diathesis or coagulopathy, assumption of anticoagulants and drugs known to predispose to gastrointestinal ulceration and psychiatric disorders or psychological disabilities thought to adversely affect treatment compliance. Patients who underwent major surgical procedure, open biopsy, or significant traumatic injury within 4 weeks prior to study treatment start were also excluded. Pregnant or lactating patients and women with childbearing potential who lacked effective contraception were excluded.

\section{Pre-treatment evaluation}

Complete history and physical examination with blood pressure, digital rectal examination, colonoscopy with tumor biopsy, endorectal ultrasound, CT and MRI of the pelvis, abdominal CT, and chest X-ray. Complete laboratory tests included full blood counts, blood electrolytes, creatinine, urea, liver transaminases, alkaline phosphatase, total and direct bilirubin, albumin, LDH, International Normalized Ratio (INR), activated partial thromboplastin time (APTT), urine test and dipstick proteinuria. Cardiac activity was investigated by electrocardiography.

\section{Treatment}

\section{Radiotherapy}

Radiation therapy was performed by conventional fractionation over a period of 5 weeks. The daily fraction dose was $1.8 \mathrm{~Gy}$. A total dose of $45 \mathrm{~Gy}$ was given in five fractions per week over a period of 5 weeks. The treated volume included the macroscopic tumor and its potential extensions within the rectum, extending $6 \mathrm{~cm}$ above and $4 \mathrm{~cm}$ below the tumor margins and extended $3 \mathrm{~cm}$ laterally and antero-posteriorly around the macroscopic limits of the tumor, the mesorectum and the perirectal lymph nodes. The anus was not irradiated unless the tumor extended into the anal canal. All the patients received a total dose of 45 Gy in daily fractions of $1.8 \mathrm{~Gy}$ (days 1-5/week) calculated at the International Commission of Radiation Units reference point, at the intersection of the central axes of the three or four beams. A boost to the macroscopic tumor with a $2-\mathrm{cm}$ peripheral margin was planned up to $50.4 \mathrm{~Gy}$ in further three fractions.

\section{Bevacizumab and capecitabine}

Bevacizumab was administered by intravenous infusion at the dose of $5 \mathrm{mg} / \mathrm{kg}$ every 2 weeks for 4 courses, with the first infusion given 2 weeks before the concurrent administration of capecitabine and radiation therapy.

Capecitabine was orally administered at the fixed dose of $825 \mathrm{mg} / \mathrm{m}^{2}$ twice a day (interval about $12 \mathrm{~h}$ ), within $30 \mathrm{~min}$ after the end of a meal, continuously for 5.5 weeks, starting from the first day of radiotherapy (days $1 \rightarrow 38$ ). The first daily dose was administered approximately $2 \mathrm{~h}$ before RT, with the second dose administered $12 \mathrm{~h}$ later.

Patients were monitored biweekly regarding history, clinical examination with PS, blood count, INR/APTT and biochemistry, including liver function.

The National Cancer Institute Common Toxicity Criteria, version 3.0, was used to grade toxicity. The schedule of bevacizumab was modified in the event of Grade 2-3 of thrombotic, hemorrhagic, proteinuric, hypertensive and allergic adverse events, as pre-specified in details. The drug was withdrawn in the case a patient experienced Grade 4 toxicity, Grade 3 toxicity not resolved to Grade 1 within 4 weeks, arterial thromboembolic events or gastrointestinal perforation.

The doses of capecitabine were adjusted for adverse events. In brief, in case of capecitabine Grade $\geq 2$ toxicity, the drug was interrupted and appropriate symptomatic treatment was administered. When the toxicity resolved to Grade $0-1$, treatment was continued at $75 \%$ of the original dose in case of the first appearance of the respective toxicity and at $50 \%$ of the starting dose in case of the second appearance. In case of Grade 3 toxicity capecitabine was withheld until the toxicity resolved to Grade $0-1$, and then continued at $75 \%$ of original dose. If radiotherapy caused local Grade $\geq 2$ toxicity, it was withheld until toxicity resolved to Grade $0-1$, and then restarted with prophylactic treatment. If the same Grade $\geq 2$ toxicity recurred, treatment was withheld until the toxicity resolved to Grade $0-1$ and the treatment was restarted if clinically necessary with a decreased dose or longer intervals in-between radiation, with a concomitant reduction of the chemotherapy schedule as detailed above. 
If toxicity required a dosing delay or interruption of all study drugs for more than 3 weeks, the patient was withdrawn from the study. If capecitabine has to be discontinued permanently due to toxicity, the patient will be allowed to continue only with bevacizumab. If bevacizumab had to be discontinued permanently due to toxicity, the patient will be allowed to continue only with capecitabine.

The relative dose intensity of bevacizumab, capecitabine and RT was calculated as the dose intensity divided by the planned dose intensity $\times 100$.

\section{Surgery}

Within 6-8 weeks after completion of neoadjuvant therapy and at least 6 weeks after the last dose of bevacizumab, surgery with total mesorectal excision was performed. Surgery was performed with curative intent with at least a minimal distance of $2 \mathrm{~cm}$ between the inferior and superior margin of the tumor and the limit of resection. Assessment of the intended surgical procedure (i.e. whether sphincter preservation was deemed possible or not) was performed by the treating surgeon before start of treatment. If adjacent organs were involved, intraoperative surgery was extended to partial or total resection of adjacent pelvic organs. Resection of metastatic sites evident during surgery was allowed.

\section{Adjuvant chemotherapy}

Four to 6 weeks after radical (R0) surgery all patients were candidate to receive adjuvant chemotherapy with bolus and infusional 5-fluouracil plus leucovorin (e.g. De Gramont regimen for 12 courses-6 months of treatment). Adjuvant treatment with bevacizumab $\left(5 \mathrm{mg} / \mathrm{m}^{2}\right.$ every 14 days $)$ was at the investigator's discretion.

\section{Pathology}

The extent of residual tumor in the resected specimen was classified according to the TNM staging system of the American Joint Committee on Cancer/International Union Against Cancer. Residual tumor area (RTA) and fibrosis after preoperative therapy were assessed based on the area of cancer and fibrosis, namely the cancer area/cancer area + fibrosis area. Tumor necrosis area (TNA) was calculated as necrosis area/necrosis area + fibrosis area and was semi-quantitatively evaluated according to a 5-point rectal cancer regression grading (tumor regression grade, TRG) suggested by Mandard et al. [20]. We used for statistical purpose the arbitrary cut-off of $15 \%$ of RTA to calculate the correlations with the biomarkers tested. A pathologic complete response was defined as the absence of viable tumor cells both in the primary tumor and lymph nodes
(ypT0N0), corresponding to Mandard TRG1. Pathologic assessment was performed by an independent pathologist (AY) blinded to the clinic-pathologic data of the patient.

Translational study of predictive and prognostic biomarkers

The tested biomarkers have been evaluated by immunohistochemical assays according to the manufacturer's instruction: CD31, anti-CD31 antibody (JC70A clone, Dako Cytomation, Denmark); CD34, anti-CD34 antibody (QBend-10 clone, Dako Cytomation, Denmark); CD68, anti-CD68 antibody (Dako Cytomation, Denmark); M30, M30 antibody (Roche, Germany); VEGFR-2, anti-VEGFR2 antibody (55B11 clone, Cell Signaling, MA); Ki67, antiKi67 antibody (Invitrogen, CA); Thymidine synthase (TS), anti-TS antibody (Exalpha Biologicals, MA); Thymidine phosphorylase (TP), anti-TP antibody (Abcam, MA).

Microvessel density was assessed by using two different antibodies: anti-CD31 and anti-CD34. The number of cells positive for CD68 and M30 were counted in five different high power fields $(\times 400)$ and the average was used for scoring. The labeling index for Ki67 was calculated as the average of Ki67positive cells/all cancer cells in five different high power fields. The intensity of the staining for VEGFR-2, TP and TS was evaluated separately in cancer cells and stromal cells by comparing the slides stained with immunohistochemistry and those with hematoxilin-eosin and it was estimated on a threetiered scale, encoded as 0 (absent), 1 (weak), 2 (strong). The score of staining intensity was evaluated by three independent pathologists (AY; YM; SN).

\section{Statistical analysis}

The primary end-point of the present phase II study was to determine the ypCR rate after neoadjuvant therapy. Secondary end-points included: toxicity, the safety profile of the neoadjuvant therapy, the sphincter-saving procedure rate, clinical tumor and lymph node regression rate, local control and the disease free survival (DFS). Simon's methods will be used to calculate the sample size. Considering the optimal two stage design for phase II study, with the difference $\mathrm{p} 1-\mathrm{p} 0=15 \%$ between "standard" chemo-radiation $(\mathrm{p} 0=10 \%)$ and "new therapy" ( $\mathrm{p} 1=25 \%)$, and fixing error probabilities $(\alpha=0.05$ and $\beta=0.20$ ), the total number of patients to be enrolled was 43. The Chi-square test and Fisher's exact test was used for qualitative parameters. Statistical differences within quantitative parameters was determined by Mann-Whitney's nonparametric test. DFS and local disease free survival (local control) were assessed by using the Kaplan and Meyer's method. Multivariate survival analysis was performed using Cox's proportional hazard ratio. Results were 
considered to be statistically significant when $P<0.05$ (two-sided). Pathological compete response, residual tumor, and tumor necrosis has been analyzed in association with DFS by using the Cox regression approach.

Correlation analysis was performed by the Pearson correlation coefficient and two unpaired groups were compared by the Student's $T$ test. When the correlations between DFS and biomarkers were examined, the Log-rank test and the Cox regression approach were adopted.

\section{Results}

Patient characteristics

Forty-three patients have been enrolled at the seven Institutions involved in the study from December 2005 through May 2007. The main characteristics of the patients are listed in Table 1. Three patients had cT4 disease (7\%), 14 $(32.6 \%)$ were staged cT3N0, four patients had cT2, N+ disease $(9.3 \%)$ and overall, radiological lymph node involvement was detected in $28(65.1 \%)$ patients. A patient had unrecognized metastatic disease and died from progressive disease 14 days after the first dose of bevacizumab $(2.3 \%)$ and two patients $(4.6 \%)$ refused surgery. Forty $(93 \%)$ patients were evaluable for response and $42(97.7 \%)$ for toxicity. All the 43 patients were included in the intentto-treat analysis. Twenty-four $(55.8 \%)$ of them received adjuvant chemotherapy.

\section{Efficacy}

Central histologic examination of post surgery specimens revealed no residual cancer both at the primary tumor

Table 1 Baseline characteristics of the patients and tumors

\begin{tabular}{lcc}
\hline Median age (range) & 64 years $(28-78)$ & \\
\hline Gender & & \\
$\quad$ Male & 25 & $58.1 \%$ \\
$\quad$ Female & 18 & $41.9 \%$ \\
P.S. (ECOG) & & \\
0 & 34 & $79.1 \%$ \\
1 & 9 & $20.9 \%$ \\
cT2,N1,M0 & 4 & 9.3 \\
cT3,N0,M0 & 14 & 32.56 \\
cT3,N1,M0 & 20 & 46.51 \\
cT3,Nx,M0 & 1 & 2.33 \\
cT4,N1,M0 & 1 & 2.33 \\
cT4,N2,M0 & 1 & 2.33 \\
cTX,N2,M0 & 1 & 2.33 \\
cT4,N2,M1 & 1 & 2.33 \\
\hline
\end{tabular}

(ypT0 or Mandard TRG 1) and in lymph nodes (ypN0) in 6 out of 43 patient (14\% 95\% CI 3.60-24.31\%). In 22 $(51.2 \%)$ patients a varying percentage of cancer cells in residual area of fibrosis or necrosis were found, corresponding to ypT1-3 or Mandard TRG 2 or 3. Seven (16.3\%) of these patients had $\leq 10 \%$ of cancer cells in fibrosis/necrosis area (Table 2).

Resection with negative circumferential margins was achieved in $38(95 \%)$ out of the 40 resected patients. A T-downstaging (the post-treatment migration of stage from initial T3/4 to ypT1-2) was observed in $15(34.9 \%)$ patients. For 19 patients $(44.2 \%)$ the T parameter remained stable. Of the 28 patients with imaging detectable lymph node disease at presentation, $16(37.2 \%)$ had N-downstaging (no lymph node disease) post-treatment (Tables 2 and 3 ).

Three patients $(6.9 \%)$ developed distant metastases after the neoadjuvant treatment; two underwent surgery. Forty patients $(93 \%)$ are alive at 16.7 months of median followup (range 14-780 days). Eight patients (18.6\%) experienced relapse of disease; 5 (11.6\%) with local recurrence only; three patients $(7 \%)$ developed metastatic disease: one in the liver, one in the lung, and the last in both sites. Actuarial 3-years disease-free survival was $75.4 \%$ (pCR group 100\%, non-pCR group 70.7\%; Fig. 1). Sphinctersparing surgery was achieved in 31 patients $(72.1 \%)$.

\section{Safety}

Most of the adverse events of the neoadjuvant treatment were mild (Grade 1/2; Table 4). There was no Grade 4 or 5 toxicity. Some of the patients experienced Grade 3 toxicities, including diarrhea (3 patients, 7.14\%), neutropenia (2 patients), asthenia and hypokalemia (1 patients, respectively). Four (9.52\%) patients permanently discontinued treatment due to $\mathrm{G} 3$ adverse events, which occurred during the last week of treatment. The most frequent sideeffects were: G1/2 diarrhea (12 patients; 28.56\%), G1/2 proctitis/proctalgia (9 patients; $21.42 \%$ ). Hand-foot syndrome (HFS) was mild and transient (only 1 patient with G2 HFS). Bevacizumab-related toxicity included: hypertension in 3 patients (7.14\%; G1 = 1 patients; $\mathrm{G} 2=2$

Table 2 Overall response to the neoadjuvant treatment

\begin{tabular}{ll}
\hline Characteristics $(n=40)$ & $\begin{array}{l}\text { No. of } \\
\text { patients }(\%)\end{array}$ \\
\hline Pathologic complete response & $6(14)$ \\
Microscopic residual disease & $7(16.3)$ \\
$\quad(\leq 15 \%$ tumor area/fibrosis-necrosis area) & \\
T downstaging & $15(34.9)$ \\
N downstaging & $18(41.86)$ \\
T stable downsizing & $19(44.2)$ \\
\hline
\end{tabular}


Table 3 Migration of stage after the neoadjuvant treatment

\begin{tabular}{|c|c|c|c|c|c|c|c|c|c|}
\hline Base line & Pts no. & урT0 & ypT1 & yPT2 & урT3 & ypT4 & ypN0 & ypN1 & ypN2 \\
\hline $\mathrm{cT} 2$ & 4 & 1 & 0 & 2 & 1 & 0 & - & - & - \\
\hline cT3 & 33 & 5 & 2 & 13 & 13 & 0 & - & - & - \\
\hline cT4 & 2 & 0 & 0 & 0 & 2 & 0 & - & - & - \\
\hline cTx & 1 & 0 & 0 & 0 & 1 & 0 & & & \\
\hline cNO & 14 & - & - & - & - & - & 12 & 2 & 0 \\
\hline $\mathrm{cN}+$ & 25 & - & - & - & - & - & 18 & 5 & 2 \\
\hline $\mathrm{cNx}$ & 1 & - & - & - & - & - & 1 & & \\
\hline Total & $40 / 43$ & 6 & 2 & 15 & 17 & 0 & & & \\
\hline
\end{tabular}

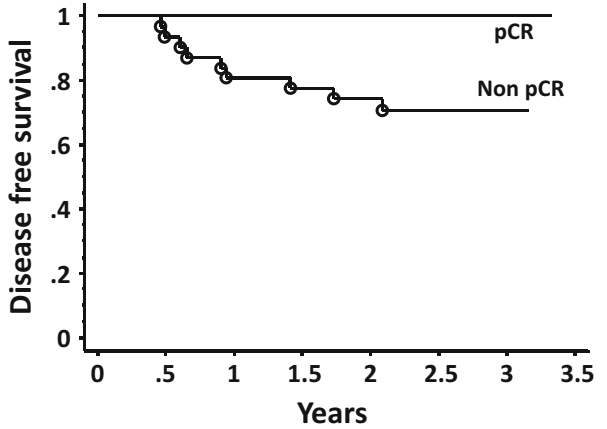

Fig. 1 Disease free survival by response to the treatment (Kaplan-Meier curve)

patients), proteinuria in 1 patient $(2.38 \%$; G2), epistaxis in 1 patient $(2.3 \%$; G2). No major hemorrhages, thromboembolic events or perforation occurred.

Overall 7 patients experienced serious adverse events, including the death of a patient with progressive disease. One patient died due bowel perforation before receiving adjuvant treatment (1 month after surgery, 81 days after the last dose of bevacizumab). Another patient presented failure to anastomosis (97 days after last bevacizumab) and a patient experienced postoperative abscess (75 days after bevacizumab). Two patients were admitted to hospital due to G3 hypokaliemia and myocardial ischemia, resolved with medical treatment. Only the latter patient was under adjuvant chemotherapy.

\section{Biomarkers of response and prognosis}

Biological correlations were performed on 27 biopsy specimens and 38 surgical specimens among the 40 patients evaluable for response with both biopsy and surgical adequate specimens available from 25 patients.

\section{Relationship between biomarkers and clinical outcomes}

No significant statistical correlation was observed between ypCR and any biomarker tested. By simple logistic regression model, pre-treatment levels of CD34-positive vessel density were associated with RTA. The patients having tumors with higher density of CD34-positive vessels as compared to those tumors with lower CD34-stained vessels were significantly associated with RTA less than $15 \%$ $(P=.0358$; Fig. 2). There was no correlation between treatment response and the pre-treatment levels of all the other markers including CD31-positive vessel density, VEGFR-2 expression both in tumor cells $(P=.42)$ and stromal cells $(P=.28)$, TP both in tumor cells $(P=.70)$ and stroma $(P=.47)$, TS both in tumor cells $(P=.99)$ and stroma $(P=.99), \operatorname{CD} 68(P=.60)$ and M30 $(P=.30)$.

When the correlations between DFS and biomarkers were examined by multiple regression model (log-rank test and Cox regression approach), no biomarker showed a statistically significant correlation with DFS.

When the association between post-treatment levels of each marker and RTA was examined, a statistically significant correlation between post-treatment Ki67 labeling indexes and levels of VEGFR-2 expression in cancer cells was found $(P<.0001$ and $P=.007$, respectively; Figs. 3, 4).

\section{Relationship between pathological response and prognosis}

The pathologic response (ypCR, residual tumor, and tumor necrosis) has been analyzed in association with DFS by using the Cox regression approach. Since no event was observed in the ypCR group, neither log-rank test nor the Cox regression analysis gave a result.

When the associations between DFS and RTA, or between DFS and TNA area was evaluated, there was a trend for a correlation between DFS and RTA by the Cox regression analysis $(P=.12)$.

\section{Discussion}

The seminal translational study by Willet et al. [19] confirmed, for the first time, that bevacizumab exhibits antivascular effects in human rectal cancer by decreasing tumor blood perfusion, microvessel density, the interstitial 
Table 4 Main toxicity during the neoadjuvant treatment

\begin{tabular}{|c|c|c|c|c|}
\hline Side effect & Total $(\%)$ & G1 & G2 & G3 \\
\hline Abdominal pain & $1(2.38)$ & $1(2.38)$ & & \\
\hline Anorectal discomfort & $2(4.76)$ & $1(2.38)$ & $1(2.38)$ & \\
\hline Constipation & $1(2.38)$ & $1(2.38)$ & & \\
\hline Diarrhoea & $15(35.7)$ & $7(16.66)$ & $5(11.9)$ & $3(7.14)$ \\
\hline Haemorrhoids & $1(2.38)$ & $1(2.38)$ & & \\
\hline Nausea & $1(2.38)$ & $1(2.38)$ & & \\
\hline Perianal erythema & $1(2.38)$ & & $1(2.38)$ & \\
\hline Proctalgia & $5(11.9)$ & $2(4.76)$ & $3(7.14)$ & \\
\hline Proctitis & $4(9.52)$ & $2(4.76)$ & $2(4.76)$ & \\
\hline Rectal haemorrhage & $1(2.38)$ & $1(2.38)$ & & \\
\hline Rectal tenesmus & $2(4.76)$ & $2(4.76)$ & & \\
\hline Asthenia & $4(9.52)$ & $2(4.76)$ & $1(2.38)$ & $1(2.38)$ \\
\hline Fatigue & $3(7.14)$ & $2(4.76)$ & $1(2.38)$ & \\
\hline Cystitis & $3(7.14)$ & $2(4.76)$ & $1(2.38)$ & \\
\hline Hyperuricaemia & $1(2.38)$ & $1(2.38)$ & & \\
\hline Hypokalaemia & $2(4.76)$ & & $1(2.38)$ & $1(2.38)$ \\
\hline Dysuria & $3(7.14)$ & $2(4.76)$ & $1(2.38)$ & \\
\hline Proteinuria & $1(2.38)$ & & $1(2.38)$ & \\
\hline Haematuria & $2(4.76)$ & & $2(4.76)$ & \\
\hline Pollakiuria & $1(2.38)$ & $1(2.38)$ & & \\
\hline Strangury & $2(4.76)$ & & $2(4.76)$ & \\
\hline $\begin{array}{l}\text { Vulvovaginal burning } \\
\text { sensation }\end{array}$ & $1(2.38)$ & & $1(2.38)$ & \\
\hline Hypoxia & $1(2.38)$ & $1(2.38)$ & & \\
\hline Dermatitis & $1(2.38)$ & $1(2.38)$ & & \\
\hline Dermatitis exfoliative & $1(2.38)$ & & $1(2.38)$ & \\
\hline Hypertension & $3(7.14)$ & $1(2.38)$ & $2(4.76)$ & \\
\hline Anaemia & $1(2.38)$ & $1(2.38)$ & & \\
\hline Leukopenia & $3(7.14)$ & $2(4.76)$ & $1(2.38)$ & \\
\hline Neutropenia & $6(14.28)$ & $3(7.14)$ & $1(2.38)$ & $2(4.76)$ \\
\hline Epistaxis & $1(2.38)$ & & $1(2.38)$ & \\
\hline
\end{tabular}

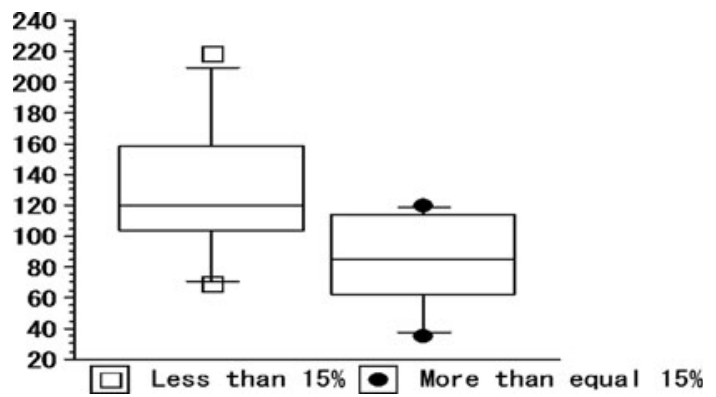

Fig. 2 Using the cut-off value of $15 \%$ for residual tumor area, the patients with values $<15 \%$ showed a statistically significant higher CD-34 vessel staining

fluid pressure and the number of circulating endothelial cells, all these parameters being associated with tumor stabilization or regression.

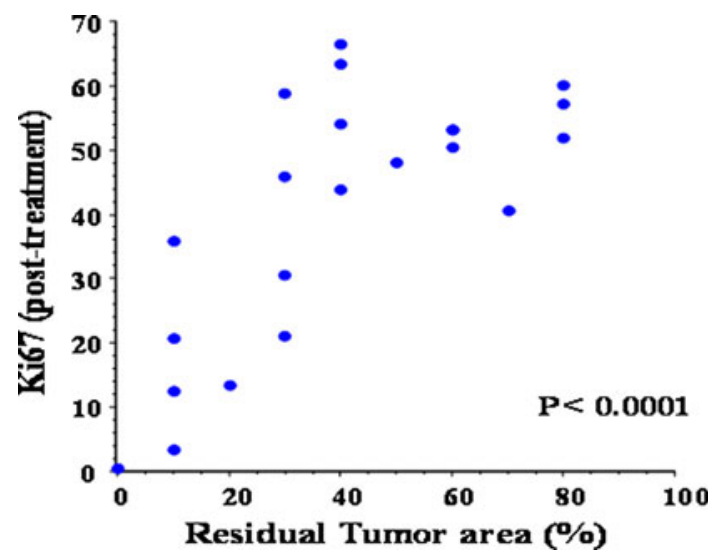

Fig. 3 Correlation between post-treatment Ki67 level and residual tumor area

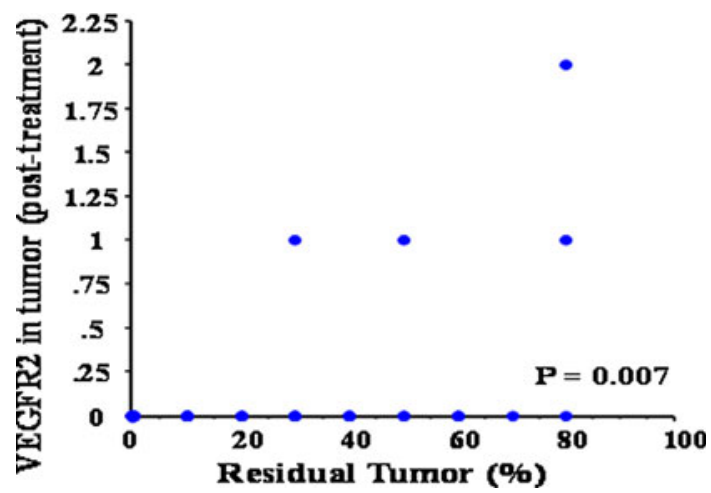

Fig. 4 Correlation between post-treatment tumor VEGFR-2 levels and residual tumor area

A second phase I-II study was performed by Willet et al. [20] to assess the safety and efficacy of neoadjuvant bevacizumab with standard chemoradiotherapy in a cohort of 32 patients with LARC. The Authors demonstrated that all the patients achieved some degree of local response and a ypCR in 5 cases (16\%). All the patients underwent a RO resection [20]. When the Authors correlated the changes in biomarkers with treatment outcome they found that VEGFR-1 pretreatment inversely correlated with tumor regression and that circulating VEGF and IL-6 levels predicted enhanced efficacy of the combined therapy.

In the present study histologic examination of postsurgical specimens revealed no residual disease in both the primary tumor and perirectal lymph nodes in 6 of 43 patients. A low percentage of cancer cells was found in the residual area of fibrosis or necrosis in 22 patients (51.2\%), corresponding to $\mathrm{ypT}_{1-3}$ or Mandard classification TRG 2 or 3 , with 7 cases $(16.3 \%$ ) among these patients with $\leq 15 \%$ of cancer cells in fibrosis/necrosis area. During the followup 8 patients experienced recurrent disease, 5 of whom with local relapse alone. Sphincter-sparing surgery was achieved in 31 cases $(72.1 \%)$. Regarding safety, most of 
the adverse side effects of the combined neoadjuvant therapy were mild with only 5 patients who experienced Grade 3 toxicity (diarrhea, neutropenia, and hypokalemia).

Similar results have been recently reported by Velenik et al. [21] and by Crane et al. [22] in a small cohort of 25 patients by using a slightly higher dosage of capecitabine and the same schedule of bevacizumab and radiation therapy as in our study. Furthermore, comparable ypCR rates have been obtained in a phase I dose-escalation study by Czito et al. [23] and by Kennecke et al. [24] in a phase II trial, where concurrent oxaliplatin, capecitabine and bevacizumab combined with radiation therapy were evaluated.

Noguè et al. [25] reported the results of a neoadjuvant phase II study with four cycles of oxaliplatin, capecitabine (XELOX regimen) and bevacizumab followed by capecitabine, bevacizumab and concomitant radiation therapy. Using the above intensive therapy the Authors reported a ypCR rate of $36 \%$ in 45 evaluable patients.

In a subset of 27 cases we obtained sufficient pre- and post-treatment pathologic material to perform correlations between the biomarkers tested and the efficacy of combined therapy. We used the two panendothelial markers CD31 and CD34 because human vascular endothelium is antigenically heterogeneous [26] and each antibody gives different staining information. In particular, CD31 is a glycoprotein expressed during the differentiation of myelomonocitic cells which is identical to the PECAM-1 molecule present in endothelial cells of blood capillaries and lymphatic microvessels. It is preferentially detected within intercapillary junctions. The expression of CD34, recognizing a transmembrane glycoprotein of $115 \mathrm{kD}$, is mostly confined to abluminal endothelium microprocesses and may indicate early endothelial cell sprouting and migration [27]. No statistically significant correlation was observed between ypCR and any biomarker tested. Pretreatment levels of CD34 vessel density were significantly associated to RTA. The patients with RTA $\leq 15 \%$ showed higher density of CD34-positive vessels as compared to those with larger RTA $(P=.0358)$, suggesting that the more vascularized tumors positive for CD34 staining responded better to neoadjuvant treatment. On the contrary, MVD assessed by using the anti-CD31 antibody was not statistically associated to RTA. These different results may be explained by the diverse sensitivity and specificity of the two panendothelial markers used in the identification of endothelial cells within tumor microvessels [28].

We further analyzed the correlation of post-treatment biomarkers and RTA. A significant correlation was observed of Ki-67 labeling index and RTA $(P<.0001)$ in agreement with the results by Jacob et al. [29]. It appears plausible that the persistence of a fraction of high-proliferative cells in a larger RTA might be representative of resistant clones of cells and therefore, of a more aggressive phenotype. However, a longer follow up is needed to demonstrate whether the persistence of a variable percentage of high-proliferative cells influences or not either local relapse rate or survival of patients. Furthermore, low levels of VEGFR-2 expression in cancer cells after therapy were associated to RTA $(P=.007)$. Zlobec et al. [30] in a cohort of 104 patients with LARC treated with preoperative radiotherapy alone found that the combined analysis of VEGF and EGFR was predictive of ypCR, with the subgroup of cases with VEGF-positive and EGFR-negative tumors being unresponsive to radiation therapy. However, in such a translational study no patient was treated with bevacizumab.

We assessed tumor associated macrophages (TAMs) because of their role in tumor angiogenesis and sensitivity to anticancer treatments [31]. Steidl et al. [32] suggested that the expression of CD68, a pan-macrophages endosomal glycoprotein, is the best biomarker for macrophages. In our study no association between CD68 stained cells and outcome was demonstrated. This may be due to the complex functions of TAMs that comprise phenotypically and functionally different cell subsets in human cancers. In fact, there is in literature conflicting evidence on the correlation of macrophage numbers with response to treatments and prognosis in different tumor types [33]. Furthermore, we evaluated apoptosis using the M30 antibody and we did not observe a significant association of M30 staining with treatment response. However, this negative result may be attributable to the limited number of cases evaluated.

Kocakoya et al. [34] demonstrated in a cohort of 55 patients affected by LARC and treated with neoadjuvant capecitabine and radiotherapy that TP and TS mRNA were induced by therapy in both responders and non-responders. In our study both TP and TS were not predictive of responsiveness in accordance with the study performed by Boskos et al. [35] in patients with LARC treated with preoperative capecitabine and radiotherapy.

In conclusion, the results of this single-arm phase II study show that such a schedule is active and safe. However, the measures of response did not suggest that bevacizumab adds significant improvement of ypCR rate or the long-term local control over standard neoadjuvant fluoropyrimidine-RT schedules.

A number of open questions remain to be elucidated on the optimal use of antiangiogenic agents in combination with chemotherapy and radiation therapy, in particular regarding the optimal timing of delivery of bevacizumab and the key problem to identify predictive biomarkers of activity capable to select the subgroup of patients who are more likely to gain benefit of antiangiogenic therapy [36] Long-term follow-up studies regarding the impact of such a therapeutic strategy on DFS and OS and regarding 
sub-acute, or chronic morbidity of neoadjuvant bevacizumab are needed. Finally, the analysis of the predictive biomarkers tested in our study, by using low-cost and feasible methods, did not reveal any significant association with ypCR.

Acknowledgments The study was supported in part by Roche S.p.A., Italy. We are indebted to the following investigators who participated in the study: Monica Lencioni, Oncologia Medica Ospedale S. Chiara, Pisa; Roberto Murialdo, Dipartimento Medicina Interna Università di Genova; Cristina Granetto, Oncologia Medica, Ospedale S. Croce \& Carle, Cuneo; Angelo Martignetti, Dipartimento Oncologia AUSL7, Siena.

\section{References}

1. Cammà $\mathrm{C}$, Giunta $\mathrm{M}$, Fiorica $\mathrm{F}$, Pagliaro L, Craxì $\mathrm{A}$, Cottone $\mathrm{M}$ (2000) Preoperative radiotherapy for resectable rectal cancer: a meta-analysis. JAMA 284:1008-1015

2. Colorectal Cancer Collaborative Group (2001) Adjuvant radiotherapy for rectal cancer: a systematic overview of 8,507 patients from 22 randomised trials. Lancet 358:1291-1304

3. Bosset JF, Colette L, Calais G, Mineur L, Maingon P, RadosevicJelic L et al (2006) Chemotherapy with preoperative radiotherapy in rectal cancer. N Engl J Med 355:1114-1123

4. Gerard JP, Conroy T, Bonnetain F, Bouché O, Chapet O, ClosonDejardin MT et al (2006) Preoperative radiotherapy, with or without concurrent 5fluorouracil and leucovorin in T3-T4 rectal cancers: results of the FFCD 9203 trial. J Clin Oncol 24:4620-4625

5. Roh MS, Colangelo LH, O'Connell MJ, Yothers G, Deutsch M, Allegra CJ et al (2009) Preoperative multimodality therapy improves disease-free survival in patients with carcinoma of the rectum: NSABP R-03. J Clin Oncol 27:5124-5130

6. Sawada N, Ishikawa T, Sekiguchi F, Tanaka Y, Ishitsuka H (1999) X-ray irradiation induces thymidine phosphorylase and enhances the efficacy of capecitabine (xeloda) in human cancer xenografts. Clin Cancer Res 5:2948-2953

7. Ben-Josef E (2007) Capecitabine and radiotherapy as neoadjuvant treatment for rectal cancer. Am J Clin Oncol 30:649-655

8. Cividalli A, Ceciarelli F, Livdi E, Altavista P, Cruciani G, Marchetti P et al (2002) Radiosensitization by oxaliplatin in a mouse adenocarcinoma: influence of treatment schedule. Int $\mathrm{J}$ Radiat Oncol Biol Phys 52:1092-1098

9. Gérard JP, Azria D, Gourgou-Bourgade S, Martel-Laffay I, Hennequin C, Etienne PL et al (2010) Comparison of two neoadjuvant chemoradiotherapy regimens for locally advanced rectal cancer: results of the phase III trial ACCORD 12/0405-Prodige 2. J Clin Oncol 28:1638-1644

10. Aschele C, Cionini L, Lonardi S, Pinto C, Cordio S, Rosati S et al (2011) Primary tumor response to preoperative chemoradiation with or without oxaliplatin in locally advanced rectal cancer: pathologic results of the STAR-01 randomized Phase III trial. J Clin Oncol 29:2773-2780

11. Weiser MR (2011) Rectal cancer trials: no movement. J Clin Oncol 29:2746-2748

12. Debucquoy A, Machiels JP, McBride WH, Haustermans K (2010) Integration of epidermal growth factor receptor inhibitors with preoperative chemoradiation. Clin Cancer Res 16:2709-2714

13. Rödel C, Arnold D, Hipp M, Liersch T, Dellas K, Iesalnieks I et al (2008) Phase I-II trial of cetuximab, capecitabine, oxaliplatin, and radiotherapy as preoperative treatment in rectal cancer. Int J Radiat Oncol Biol Phys 70:1081-1086

14. Hurwitz HI, Fehrenbacher L, Hainsworth JD, Heim W, Berlin J, Holmgren $\mathrm{E}$ et al (2005) Bevacizumab in combination with fluorouracil and leucovorin: an active regimen for first-line metastatic colorectal cancer. J Clin Oncol 23:3502-3508

15. Allegra CJ, Yothers G, O'Connell MJ, Sharif S, Petrelli NJ, Colangelo LH et al (2011) Phase III trial assessing bevacizumab in stages II and III carcinoma of the colon: results of NSABP protocol C-08. J Clin Oncol 29:11-16

16. De Gramont A, Van Cutsem E, Tabernero J, Moore MJ, Cunningham D, Rivera $\mathrm{F}$ et al (2011) AVANT: results from a randomized, three-arm multinational phase III study to investigate bevacizumab with either XELOX or FOLFOX4 versus FOLFOX4 alone as adjuvant treatment for colon cancer. J Clin Oncol 29(suppl 4); abstr 362

17. Willett CG, Kozin SV, Duda DG, di Tomaso E, Kozak KR, Boucher Y et al (2006) Combined vascular endothelial growth factor-targeted therapy and radiotherapy for rectal cancer: theory and clinical practice. Semin Oncol 33(Suppl 10):S35-S40

18. Willett CG, Boucher Y, di Tomaso E, Duda DG, Munn LL, Tong RT et al (2004) Direct evidence that the VEGF-specific antibody bevacizumab has antivascular effects in human rectal cancer. Nat Med 10:145-147

19. Willett CG, Duda DG, di Tomaso E, Boucher Y, Ancukiewicz M, Sahani DV et al (2009) Efficacy, safety, and biomarkers of neoadjuvant bevacizumab, radiation therapy, and fluorouracil in rectal cancer: a multidisciplinary phase II study. J Clin Oncol 27:3020-3026

20. Mandard AM, Dalibard F, Mandard JC, Marnay J, Henry-Amar M, Petiot JF et al (1994) Pathologic assessment of tumor regression after preoperative chemoradiotherapy of esophageal carcinoma. Clinicopathologic correlations. Cancer 73:2680-2686

21. Velenik V, Ocvirk J, Music M, Bracko M, Anderluh F, Oblak I et al (2011) Neoadjuvant capecitabine, radiotherapy, and bevacizumab (CRAB) in locally advanced rectal cancer: results of an open-label phase II study. Radiat Oncol 6:105-112

22. Crane CH, Eng C, Feig BW, Das P, Skibber JM, Chang GJ et al (2010) Phase II trial of neoadjuvant bevacizumab, capecitabine, and radiotherapy for locally advanced rectal cancer. Int J Radiat Oncol Biol Phys 76:824-830

23. Czito BG, Bendell JC, Willett CG, Morse MA, Blobe GC, Tyler DS et al (2007) Bevacizumab, oxaliplatin, and capecitabine with radiation therapy in rectal cancer: phase I trial results. Int J Radiat Oncol Biol Phys 68:472-478

24. Kennecke H, Berry S, Wong R, Zhou C, Tankel K, Easaw J et al (2011) Pre-operative bevacizumab, capecitabine, oxaliplatin and radiation among patients with locally advanced or low rectal cancer: a phase II trial. Eur J Cancer (in press)

25. Nogué M, Salud A, Vicente P, Arriví A, Roca JM, Losa F et al (2011) Addition of bevacizumab to XELOX induction therapy plus concomitant capecitabine-based chemoradiotherapy in magnetic resonance imaging-defined poor-prognosis locally advanced rectal cancer: the AVACROSS study. Oncologist $16: 614-620$

26. Kuzu I, Bicknell R, Harris AL, Jones M, Gatter KC, Mason DY (1992) Heterogeneity of vascular endothelial cells with relevance to diagnosis of vascular tumours. J Clin Pathol 45:143-148

27. Sauter B, Foedinger D, Sterniczky B, Wolff K, Rappersberger K (1998) Immunoelectron microscopic characterization of human dermal lymphatic microvascular endothelial cells. Differential expression of CD31, CD34, and type IV collagen with lymphatic endothelial cells versus blood capillary endothelial cells in normal human skin, lymphangioma, and hemangioma in situ. J Histochem Cytochem 46:165-176 
28. Miettinen M, Lindenmayer AE, Chaubal A (1994) Endothelial cell markers CD31, CD34, and BNH9 antibody to $\mathrm{H}$ - and Y-antigens - evaluation of their specificity and sensitivity in the diagnosis of vascular tumors and comparison with von Willebrand factor. Mod Pathol 7:82-90

29. Jakob C, Liersch T, Meyer W, Becker H, Baretton GB, Aust DE (2008) Predictive value of Ki67 and p53 in locally advanced rectal cancer: correlation with thymidylate synthase and histopathological tumor regression after neoadjuvant 5-FU-based chemoradiotherapy. World J Gastroenterol 14:1060-1066

30. Zlobec I, Vuong T, Compton CC, Lugli A, Michel RP, Hayashi S et al (2008) Combined analysis of VEGF and EGFR predicts complete tumour response in rectal cancer treated with preoperative radiotherapy. Br J Cancer 98:450-456

31. Jinushi M, Chiba S, Yoshiyama H, Masutomi K, Kinoshita I, Dosaka-Akita H et al (2011) Tumor-associated macrophages regulate tumorigenicity and anticancer drug responses of cancer stem/initiating cells. Proc Natl Acad Sci USA 108:12425-12430

32. Steidl C, Lee T, Shah SP, Farinha P, Han G, Nayar T et al (2010) Tumor-associated macrophages and survival in classic Hodgkin's lymphoma. N Engl J Med 362:875-885
33. Bingle L, Brown NJ, Lewis CE (2002) The role of tumourassociated macrophages in tumour progression: implications for new anticancer therapies. J Pathol 196:254-265

34. Kocakova I, Svoboda M, Kubosova K, Chrenko V, Roubalova E, Krejci E et al (2007) Preoperative radiotherapy and concomitant capecitabine treatment induce thymidylate synthase and thymidine phosphorylase mRNAs in rectal carcinoma. Neoplasma $54: 447-453$

35. Boskos CS, Liacos C, Korkolis D, Aygerinos K, Lamproglou I, Terpos E et al (2010) Thymidine phosphorylase to dihydropyrimidine dehydrogenase ratio as a predictive factor of response to preoperative chemoradiation with capecitabine in patients with advanced rectal cancer. J Surg Oncol 102:408-412

36. Zhang D, Hedlund E, Lim S, Chen F, Zhang Y, Sun B et al (2011) Antiangiogenic agents significantly improve survival in tumorbearing mice by increasing tolerance to chemotherapy-induced toxicity. Proc Natl Acad Sci USA 108:4117-4122 